\title{
Prevalence and Prognostic Role of PIK3CA/AKT1 Mutations in Chinese Breast Cancer Patients
}

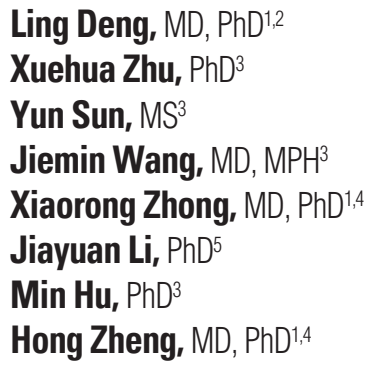

${ }^{1}$ Laboratory of Molecular Diagnosis of Cancer, Clinical Research Center for Breast,

State Key Laboratory of Biotherapy, National Collaborative Innovation Center for Biotherapy, West China Hospital, Sichuan University, Chengdu, ${ }^{2}$ Laboratory of Pathology, West China Hospital, Sichuan University, Chengdu, ${ }^{3}$ Dizal (Jiangsu) Pharmaceutical Co., Ltd., Shanghai, ${ }^{4}$ Cancer Center, West China Hospital, Sichuan University, Chengdu, ${ }^{5}$ Department of Epidemiology and Bio-Statistics,

West China School of Public Health,

Sichuan University, Chengdu, China

\section{Correspondence: $\mathrm{Min} \mathrm{Hu}$, $\mathrm{PhD}$}

Dizal (Jiangsu) Pharmaceutical Co., Ltd.

Shanghai 201203, China

Tel: 86-21-6109-7820

Fax: 86-21-5838-7361

E-mail: min.hu.jx@hotmail.com

Co-correspondence: Hong Zheng, MD, PhD Laboratory of Molecular Diagnosis of Cancer, Clinical Research Center for Breast,

West China Hospital, Sichuan University,

37 Guoxuexiang, Wuhou District, Chengdu,

Sichuan 610041, China

Tel: 86-28-8542-2685

Fax: 86-28-8542-2685

E-mail: hzheng@scu.edu.cn

Received December 20, 2017

Accepted March 12, 2018

Published Online March 15, 2018

*Ling Deng and Xuehua Zhu contributed equally to this work.

\section{Purpose}

The prevalence of PIK3CA in Chinese breast cancer patients may be underestimated. Therefore, we investigated the distribution of somatic PIK3CA/AKT1 mutations in Chinese breast cancer patients and explored their roles in tumor phenotypes and disease prognosis.

\section{Materials and Methods}

Tumors from 507 breast cancer patients were prospectively collected from the West China Hospital between 2008 and 2013. Whole exons of AKT1 and PIK3CA were detected in freshfrozen tumors using next-generation sequencing, and correlations between PIK3CA/AKT1 mutations and clinicopathological features were analyzed.

\section{Results}

The AKT1 mutation was found in 3.6\% (18/507) of patients. Tumors from patients that carried the AKT1 mutation were estrogen receptor (ER)+/progesterone receptor (PR)+/human epidermal growth factor receptor 2 (HER2)- and were more likely to have high expression levels of Ki67. The prevalence of the PIK3CA mutation was $46.5 \%$ (236/507), and 35 patients carried two or three variants of the PIK3CA gene. PIK3CA mutations were associated with ER+/PR+/HER2- status. The prognosis of patients with one mutation in PIK3CA (or PIK3CA/AKT1) was not significantly different than that of patients with wild-type PIK3CA (or PIK3CA/AKT1), while patients with two or three variants in PIK3CA (or PIK3CA/AKT1) exhibited poorer outcomes in the entire group and in all three subgroups (ER+, HER2-, Ki67 high), particularly with respect to overall survival.

\section{Conclusion}

A high frequency of somatic PIK3CA mutations was detected in Chinese breast cancer patients. In addition to the mutation frequency, the tumor mutational burden of the PIK3CA and AKT1 genes should also be of concern, as they may be associated with poor prognosis.

Key words

PIK3CA, AKT1, Breast neoplasms, Prognosis 


\section{Introduction}

The phosphoinositide 3-kinase (PI3K) signaling pathway, which promotes cancer cell growth and metabolism, is the most frequently mutated pathway in breast cancer. This pathway is associated with failure of clinical treatments, such as anti-human epidermal growth factor receptor 2 (HER2) therapy and endocrine therapy, and chemotherapy resistance $[1,2]$. Clinical development of several pan-PI3K inhibitors, PI3K $\alpha$ subunit-specific inhibitors, PI3K $\beta$ subunitspecific inhibitors, mammalian target of rapamycin (mTOR) inhibitors, and PI3K/mTOR dual inhibitors is currently underway. However, the biomarkers to evaluate the efficacy of these agents still need to be elucidated.

According to The Cancer Genome Atlas network (TCGA), the percentage of PIK3CA mutations is $34.18 \%$, and the percentage of $A K T 1$ mutations is $3.14 \%$ [3]. Reports of the prevalence of PIK3CA mutation varied among studies $(15.6 \%$ $47.6 \%$ ) [4-16], and the percentage PIK3CA mutation is between $15.6 \%$ and $28.3 \%$ in Chinese breast cancer patients $[10,11,14]$. These mutation frequencies may be underestimated. One possible reason is that most studies detected several exons with hotspot mutations via Sanger sequencing, spectrometry genotyping, or hydrolysis probe assays [4-13]. In the BOLERO-2 trial, tumor samples from 302 patients were analyzed via next-generation sequencing (NGS). In this trial, the percentage of PIK3CA mutations was $47.6 \%$, which is within the range of the most frequently mutated genes in breast cancer [15]. Another possible reason is that some studies used formalin-fixed, paraffin-embedded (FFPE) tissues $[7,10,13]$, and as preservation time increases, DNA may break down and therefore may not be amplified. In a previous study, we also detected PIK3CA and AKT1 hotspot mutations using FFPE tissues from 288 breast cancer patients via Sanger sequencing. However, the amplification failure rate of a single exon was as high as $25.7 \%$, where the frequency of PIK3CA mutation was only $15.6 \%$ [10]. Both aforementioned reasons can lead to underestimation of the PIK3CA mutation frequency.

In this single-center observational study, we investigated the frequency of PIK3CA and AKT1 mutations in tumor tissues from 507 Chinese breast cancer patients using NGS and explored their role in tumor phenotype and disease prognosis.

\section{Materials and Methods}

\section{Study patients}

All patients pathologically diagnosed with breast cancer were prospectively registered in the Breast Cancer Information Management System (BCIMS) at the West China Hospital, Sichuan University beginning in 2008. In this study, patients who had undergone surgery between 2008 and 2013 were recruited. Patients who underwent neoadjuvant chemotherapy, lacked complete clinical information, or were unable to provide enough frozen tumor tissue were excluded from this study. Ultimately, 507 patients were eligible for the PIK3CA/AKT1 mutation test and analysis of clinical characteristics.

Clinical and pathological characteristics of 507 patients were extracted from the BCIMS (S1 Table). Immunohistochemistry (IHC) scoring for estrogen receptor (ER), progesterone receptor (PR) was conducted following the Guidelines for Testing of ER and PR in Breast Cancer [17]; IHC and fluorescence in situ hybridization scoring for human epidermal growth factor receptor-2 (HER2) was performed according to the Guidelines for HER2 Detection in Breast Cancer [18]. The subtypes of breast cancer were classified according to St. Gallen International Expert Consensus [19]. Follow-up on survival status was available until May 2017. Invasive disease-free survival (iDFS) was defined as the interval between surgery date and first relapse of cancer, death, the second primary tumor, or last follow-up [20]. Overall survival (OS) was defined as the interval between surgery date and patient death, or last follow-up. Among 498 patients with stage I-III breast cancer, 57 iDFS events and 36 OS events were observed.

\section{Targeted DNA sequencing and bioinformatics}

Freshly frozen tumor tissues were obtained from the 507 breast cancer patients. Tumor content of $\geq 50 \%$ was qualified via pathology quality control. Tumor DNA samples were screened for variants in all coding exons of the PIK3CA and AKT1 genes with a customized QIAGEN Gene Read panel, using NGS on a MiSeq system (Illumina, San Diego, CA). Whole blood or distal adjacent normal tissue were used to filter out germline variants for 24 patients in which novel PIK3CA variants were identified during interim data analysis.

After trimming adapters and low-quality bases (sequencing quality cutoff at Q30), reads were mapped to Human B37.3. Genomic variants were organized using the following criteria: reads uniquely mapped to the genome; mapping quality $>15$; number of mutated reads $\geq 5$, and mutant allele 
Table 1. PIK3CA and AKT1 mutations in 507 breast cancer patients

\begin{tabular}{llclcc|}
\hline Gene & Mutation type & Occurrence & Status & Hot-spot mutation & Frequency (\%) \\
\hline AKT1 & AKT1_E17K & 17 & Tumor & Yes & 3.4 \\
& AKT1_C77F & 1 & Tumor & No & 0.2 \\
\hline PIK3CA ${ }^{\text {a) }}$ & PIK3CA_H1047R & 121 & Tumor & Yes & 23.9 \\
& PIK3CA_E545K & 50 & Tumor & Yes & 9.9 \\
& PIK3CA_H1047L & 19 & Tumor & Yes & 37.5 \\
& PIK3CA_E542K & 14 & Tumor & Yes & 27.6 \\
& PIK3CA_N345K & 8 & Tumor & No & 1.6 \\
& PIK3CA_E726K & 7 & Tumor & No & 1.4 \\
& PIK3CA_C420R & 5 & Tumor & No & 1.0 \\
& PIK3CA_G118D & 3 & Tumor & No & 0.6 \\
\hline & PIK3CA_E365K & 2 & Tumor & No & 0.4 \\
\hline & PIK3CA_P471L & 2 & Tumor & No & 0.4 \\
\hline & PIK3CA_Q546K & 2 & Tumor & No & 0.4 \\
& PIK3CA_T1025A & 2 & Tumor & No & 0.4 \\
\hline & PIK3CA_G1049R & 2 & Tumor & No & 0.4 \\
\hline
\end{tabular}

a)36 additional PIK3CA variants (tumor status) were identified with 1 occurrence: PIK3CA_C420DEL, PIK3CA_D454DEL, PIK3CA_D454N, PIK3CA_D527N, PIK3CA_D830H, PIK3CA_E103DEL, PIK3CA_E418K, PIK3CA_E545D, PIK3CA_E545Q, PIK3CA_E600K, PIK3CA_E674Q, PIK3CA_E722Q, PIK3CA_E798K, PIK3CA_E81K, PIK3CA_E970K, PIK3CA_E9DE, PIK3CA_G364R, PIK3CA_H1065L, PIK3CA_HC491DEL, PIK3CA_I102F, PIK3CA_K111E, PIK3CA_K485N, PIK3CA_ KI111N, PIK3CA_L422W, PIK3CA_M16I, PIK3CA_N1044I, PIK3CA_N1068INS, PIK3CA_N345I, PIK3CA_p.Cys472_ Leu473insPheGlu, PIK3CA_P104DEL, PIK3CA_P449DEL, PIK3CA_Q546E, PIK3CA_Q75E, PIK3CA_R115L, PIK3CA_T1052K PIK3CA_V101DEL.

frequency $\geq 0.01$. Variants were then manually verified in the Genome Browser.

\section{Statistical analysis}

Two-tailed t test, chi-square test, and Fisher exact test were performed to assess significance of the association between variables (PIK3CA and AKT mutations) and clinical characteristics (SPSS ver. 20, IBM Corp., Armonk, NY). The KaplanMeier survival curve was drawn and tested by log-rank test to evaluate the differences in iDFS and OS between the variables. The effect of mutations on prognosis was analyzed by Cox multivariate risk models. A p-value of $<0.05$ was considered statistically significant.

\section{Ethical statement}

This study was approved by the Clinical Test and Biomedical Ethics Committee of West China Hospital, Sichuan University. Written informed consent was provided by all patients.

\section{Results}

\section{Prevalence and characteristics of PIK3CA and AKT mutations}

The prevalence of PIK3CA and AKT1 mutations is shown in Table 1. AKT1 mutation was found in 18 patients (3.6\%), and the percentage of hotspot mutations for AKT1_E17K was $3.4 \%$ (17/507). PIK3CA mutation was detected in $236(46.5 \%)$ patients. PIK3CA hotspot mutations (H1047R/L, E545K, E542K) were found in $39.4 \%$ of breast cancer patients (200/ 507), with H1047R ranking the highest. These hotspot mutations account for $84.7 \%$ of breast cancer patients $(200 / 236)$ who carry somatic PIK3CA variants. There were 273 mutations of PIK3CA in total. Thirty-five patients carried two or three mutations in PIK3CA (S2 Table), and 33 had concurrent hotspot mutations with 1 or 2 non-hotspot mutations. AKT1 mutations (3 AKT1_E17K and 1 AKT1_C77F) were concurrent with PIK3CA_H1047R mutation in four patients. When AKT1 and PIK3CA mutations were combined based on mutant burden, 39 patients carried two or three mutations (2MTs) (S2 Table), 211 patients had one mutation (1MT), and 257 patients carried wild-type (WT) PIK3CA and AKT1. The clinical and pathological features of patients with PIK3CA and 
Table 2. The clinical and pathological features of patients with PIK3CA and $A K T 1$ mutations

\begin{tabular}{|c|c|c|c|c|c|c|c|c|}
\hline \multirow{2}{*}{ Characteristic } & \multirow{2}{*}{$\frac{\begin{array}{c}A K T 1 \\
\text { mutation }\end{array}}{\begin{array}{c}\text { MT } \\
(\mathrm{n}=18)\end{array}}$} & \multirow{2}{*}{ p-value } & \multicolumn{2}{|c|}{ PIK3CA mutation } & \multirow{2}{*}{ p-value } & \multicolumn{2}{|c|}{$\begin{array}{c}\text { PIK3CA/AKT1 } \\
\text { mutation burdens }\end{array}$} & \multirow{2}{*}{ p-value } \\
\hline & & & $\begin{array}{c}\text { Hotspot } \\
\text { MT (n=200) }\end{array}$ & $\begin{array}{l}\text { Non-hotspot } \\
\text { MT (n=36) }\end{array}$ & & $\begin{array}{l}\text { 2MTs } \\
(n=39)\end{array}$ & $\begin{array}{c}1 \mathrm{MT} \\
(\mathrm{n}=211)\end{array}$ & \\
\hline Age (yr) & $50.9 \pm 9.3$ & $0.72^{\mathrm{a})}$ & $50.8 \pm 10.1$ & $51.2 \pm 8.3$ & $0.24^{\mathrm{b})}$ & $50.6 \pm 9.4$ & $50.9 \pm 9.9$ & $0.20^{c)}$ \\
\hline Menopausal status & & $0.60^{\mathrm{a})}$ & & & $0.92^{\mathrm{b})}$ & & & $0.92^{c)}$ \\
\hline Premenopausal & $9(50.0)$ & & $88(44.0)$ & $17(47.2)$ & $0.720^{\mathrm{d})}$ & $17(43.6)$ & $95(45.0)$ & $0.856^{\mathrm{e})}$ \\
\hline Postmenopausal & $9(50.0)$ & & $112(56.0)$ & $19(52.8)$ & & $22(56.4)$ & $116(55.0)$ & \\
\hline Breast cancer family history & & $0.53^{\mathrm{a})}$ & & & $0.29^{\mathrm{b})}$ & & & $0.79^{c)}$ \\
\hline Yes & $1(5.6)$ & & $4(2.0)$ & $2(5.6)$ & $0.225^{\mathrm{d})}$ & 0 & $7(3.3)$ & $0.600^{\mathrm{e})}$ \\
\hline No & $17(94.4)$ & & 198 (98.9) & $34(94.4)$ & & $39(100)$ & $204(96.7)$ & \\
\hline Pathological type & & $0.59^{a)}$ & & & $0.68^{\mathrm{b})}$ & & & $0.98^{c)}$ \\
\hline DCIS & 0 & & $1(0.5)$ & $1(2.8)$ & $0.382^{\mathrm{d})}$ & 0 & $2(0.9)$ & $1.0^{\mathrm{e})}$ \\
\hline IDC & $17(94.4)$ & & $192(96.0)$ & $34(94.4)$ & & $38(97.4)$ & $201(95.3)$ & \\
\hline Other IBC & $1(5.6)$ & & $7(3.5)$ & $1(2.8)$ & & $1(2.6)$ & $8(3.8)$ & \\
\hline Tumor grade & & $0.06^{\mathrm{a})}$ & & & $0.14^{\mathrm{b})}$ & & & $0.04^{\mathrm{c})}$ \\
\hline 1 & $1(5.6)$ & & $10(5.0)$ & $1(2.8)$ & $0.149^{\mathrm{d})}$ & $3(7.7)$ & $8(3.8)$ & $0.066^{\mathrm{e})}$ \\
\hline 2 & $10(55.6)$ & & $67(33.5)$ & $9(25.0)$ & & $19(48.7)$ & $64(30.3)$ & \\
\hline 3 & $7(38.9)$ & & $108(54.0)$ & $26(72.2)$ & & $15(38.5)$ & $126(59.7)$ & \\
\hline Unknown & 0 & & $15(7.5)$ & 0 & & $2(5.1)$ & $13(6.2)$ & \\
\hline T stage & & $0.08^{\text {a) }}$ & & & $0.64^{\mathrm{b})}$ & & & $0.59^{c)}$ \\
\hline Tis/T1 & $9(50.0)$ & & $70(35.0)$ & $10(27.8)$ & $0.395^{\mathrm{d})}$ & $11(28.2)$ & 75 (35.5) & $0.495^{\mathrm{e})}$ \\
\hline $\mathrm{T} 2$ & $7(38.9)$ & & $118(59.0)$ & $24(66.7)$ & & $26(66.7)$ & $122(57.8)$ & \\
\hline T3 & 0 & & $7(3.5)$ & 0 & & 0 & $7(3.3)$ & \\
\hline $\mathrm{T} 4$ & $2(11.1)$ & & $5(2.5)$ & $2(5.6)$ & & $2(5.1)$ & $7(3.3)$ & \\
\hline $\mathrm{Tx}$ & 0 & & 0 & 0 & & 0 & 0 & \\
\hline Lymph node involvement & & $0.90^{\mathrm{a})}$ & & & $0.46^{\mathrm{b})}$ & & & $0.56^{c)}$ \\
\hline No & $9(50.0)$ & & $95(47.5)$ & $13(36.1)$ & $0.436^{\mathrm{d})}$ & $15(38.5)$ & $99(46.9)$ & $0.713^{\mathrm{e})}$ \\
\hline N1 & $7(38.9)$ & & $66(33.0)$ & $17(47.2)$ & & $17(43.6)$ & $72(34.1)$ & \\
\hline N2 & $1(5.6)$ & & $25(12.5)$ & $4(11.1)$ & & 4 (10.3) & $26(12.3)$ & \\
\hline N3 & $1(5.6)$ & & $14(7.0)$ & $2(5.6)$ & & $3(7.7)$ & $14(6.6)$ & \\
\hline Metastasis & & $0.17^{\mathrm{a})}$ & & & $0.13^{\mathrm{b})}$ & & & $0.598^{\mathrm{c})}$ \\
\hline M0 & $17(94.4)$ & & $200(100)$ & $36(100)$ & & $39(100)$ & $210(99.5)$ & \\
\hline M1 & $1(5.6)$ & & 0 & 0 & & 0 & $1(0.5)$ & \\
\hline Molecular subtypes & & $0.001^{\mathrm{a})}$ & & & $0.003^{\mathrm{b})}$ & & & $<0.001^{\mathrm{c})}$ \\
\hline Luminal A & $6(33.3)$ & & $25(12.5)$ & $4(11.1)$ & $0.169^{\mathrm{d})}$ & $10(25.6)$ & $23(10.9)$ & $0.140^{\mathrm{e})}$ \\
\hline Luminal B1 & $12(66.7)$ & & $110(55.0)$ & $13(36.1)$ & & $21(53.8)$ & $112(53.1)$ & \\
\hline Luminal B2 & 0 & & $28(14.0)$ & $8(22.2)$ & & $3(7.7)$ & $33(15.6)$ & \\
\hline HER2+ & 0 & & $21(10.5)$ & $7(19.4)$ & & $3(7.7)$ & 25 (11.8) & \\
\hline $\mathrm{TN}$ & 0 & & $16(8.0)$ & $4(11.1)$ & & $2(5.1)$ & $18(8.5)$ & \\
\hline ER & & $0.001^{\mathrm{a})}$ & & & $<0.001^{\mathrm{b})}$ & & & $<0.001^{\mathrm{c})}$ \\
\hline Positive & 18 (100) & & $158(79.0)$ & $25(69.4)$ & $0.206^{\mathrm{d})}$ & $34(87.2)$ & 163 (77.3) & $0.163^{\mathrm{e}}$ \\
\hline Negative & 0 & & $42(21.0)$ & $11(30.6)$ & & 5 (12.8) & $48(22.7)$ & \\
\hline PR & & $0.001^{\mathrm{a})}$ & & & $0.013^{\mathrm{b})}$ & & & $0.005^{c)}$ \\
\hline Positive & $18(100)$ & & 147 (73.5) & $21(68.3)$ & $0.064^{\mathrm{d})}$ & $30(76.9)$ & $152(72.0)$ & $0.529^{\mathrm{e})}$ \\
\hline Negative & 0 & & $53(26.5)$ & 15 (31.7) & & $9(23.1)$ & $59(28.0)$ & \\
\hline HER2 & & $0.003^{\mathrm{a})}$ & & & $0.039^{b)}$ & & & $0.021^{\mathrm{c})}$ \\
\hline Positive & 0 & & $49(24.5)$ & 15 (41.7) & $0.033^{\mathrm{d})}$ & $6(15.4)$ & $58(27.5)$ & $0.112^{\mathrm{e})}$ \\
\hline Negative & $18(100)$ & & $151(75.5)$ & $21(58.3)$ & & $33(84.6)$ & $153(72.5)$ & \\
\hline
\end{tabular}

(Continued to the next page) 
Table 2. Continued

\begin{tabular}{|c|c|c|c|c|c|c|c|c|}
\hline \multirow{2}{*}{ Characteristic } & \multirow{2}{*}{$\frac{\begin{array}{c}A K T 1 \\
\text { mutation }\end{array}}{\begin{array}{c}\text { MT } \\
(n=18)\end{array}}$} & \multirow{2}{*}{ p-value } & \multicolumn{2}{|c|}{ PIK3CA mutation } & \multirow{2}{*}{ p-value } & \multicolumn{2}{|c|}{$\begin{array}{c}\text { PIK3CA/AKT1 } \\
\text { mutation burdens }\end{array}$} & \multirow{2}{*}{ p-value } \\
\hline & & & $\begin{array}{c}\text { Hotspot } \\
\text { MT (n=200) }\end{array}$ & $\begin{array}{l}\text { Non-hotspot } \\
\text { MT (n=36) }\end{array}$ & & $\begin{array}{l}2 \mathrm{MTs} \\
(\mathrm{n}=39)\end{array}$ & $\begin{array}{c}1 \mathrm{MT} \\
(\mathrm{n}=211)\end{array}$ & \\
\hline Ki67 & & $0.001^{\mathrm{a})}$ & & & $0.62^{b)}$ & & & $0.031^{\mathrm{c})}$ \\
\hline High & $10(55.6)$ & & $163(81.5)$ & $30(83.3)$ & $0.793^{\mathrm{d})}$ & $27(69.2)$ & 175 (82.9) & $0.046^{\mathrm{e})}$ \\
\hline Low & $8(44.4)$ & & 37 (19.5) & $6(16.7)$ & & $12(30.8)$ & $36(17.1)$ & \\
\hline
\end{tabular}

Value are presented as number (\%). MT, mutation; 2MTs, 2 or 3 mutations; $1 \mathrm{MT}, 1$ mutation; DCIS, ductal carcinoma in situ; IDC, invasive ductal carcinoma; IBC, invasive breast cancer; HER2, human epidermal growth factor receptor 2; TN, triple negative subtype; Luminal B1, luminal B-HER2 negative subtype; Luminal B2, luminal B2-HER2 positive subtype; HER2+, HER2 positive subtype; ER, estrogen receptor; PR, progesterone receptor. ${ }^{a} \mathrm{p}$-value for mutant and WT AKT1, b) $\mathrm{p}$-value for hotspot mutant, non-hotspot mutant and WT PIK3CA, c) p-value for 2MTs, 1MT and WT of PIK3CA/AKT1, d) p-value for hotspot and non-hotspot mutant PIK3CA, e) p-value for 2MTs and 1MT of PIK3CA/AKT1.

AKT1 mutations are shown in Table 2.

\section{1) AKT1 mutation}

All patients with AKT1 mutations were ER+/ PR+/HER2(Table 2). In addition, the AKT1 E17K mutation was significantly associated with Ki67 high expression, but was not associated with age at diagnosis, menopausal status, family history, pathological type, tumor grade, $\mathrm{T}$ category, lymph node stage or metastasis.

\section{2) PIK3CA mutation}

Compared to breast cancers expressing wild type PIK3CA, both hotspot and non-hotspot PIK3CA mutant cancers were more likely to be ER+, PR+, HER2- or of the luminal B (HER2 negative) subtype (Table 2). PIK3CA mutation was not associated with age at diagnosis, menopausal status, family history, pathological type, tumor grade, $\mathrm{T}$ category, lymph node stage, Ki67 expression, or metastasis. No difference was observed between patients with hotspot mutations and nonhotspot mutations, except for HER2 status.

\section{3) PIK3CA/AKT1 mutation}

When AKT1 and PIK3CA mutations were analyzed by mutation burden, cancers with 2MTs or with 1MT were more likely to be ER+, PR+, HER2-, high Ki67, or of the luminal B (HER2 negative) subtype compared with cancers with WT PIK3CA and AKT1. When 2MTs and 1MT were sub-analyzed, no difference was observed between the two subgroups except for Ki67 expression.

\section{Association of PIK3CA and AKT1 mutations with sur- vival}

Survival analysis was conducted among 296 invasive stage I-III breast cancer patients with at least a 5-year follow-up. According to the tumor mutational burden of PIK3CA/AKT1, there were 18 patients with $2 \mathrm{MTs}$, 124 cases with $1 \mathrm{MT}$ and 154 cases with WT. The baseline of these 296 patients is summarized in S3 Table. Both PIK3CA and PIK3CA/AKT1 mutations were more frequent in cancers that were ER+, HER2-, or expressing high Ki67. The Kaplan-Meier survival curve and Cox multivariate risk models were used to evaluate the survival of patients.

\section{1) Total patients ( 296 cases)}

Regarding PIK3CA mutation, patients with 1MT showed better iDFS than patients with 2MTs (Fig. 1A), with a relative hazard ratio (HR) of $0.344(\mathrm{p}=0.049)$ after adjustment (Table 3). Patients with 1MT (adjusted HR, 0.222; $\mathrm{p}=0.020$ ) or WT PIK3CA (adjusted HR, 0.221; $\mathrm{p}=0.021$ ) had better OS than patients with 2MTs (Table 3, Fig. 1B). However, there was no difference in iDFS between patients with WT and 2MTs (Fig. 1A). In addition, no difference in iDFS or OS was observed between patients with WT and 1MT of PIK3CA (Fig. 1A and B).

Regarding PIK3CA/AKT1 mutation, patients with 1MT exhibited better iDFS (adjusted HR, 0.269; $\mathrm{p}=0.011$ ) and OS (adjusted HR, 0.151; $\mathrm{p}=0.002$ ) than those with 2MTs (Table 3, Fig. 1C and D). Patients with WT PIK3CA/AKT1 exhibited a better OS than those with 2MTs (adjusted HR, 0.181; $\mathrm{p}=0.006$ ) (Table 3, Fig. 1D), but no significant difference in iDFS. While patients with 1MT in PIK3CA/AKT1 showed a trend toward better iDFS than patients with WT PIK3CA/AKT1 $(\mathrm{p}=0.054)$ 

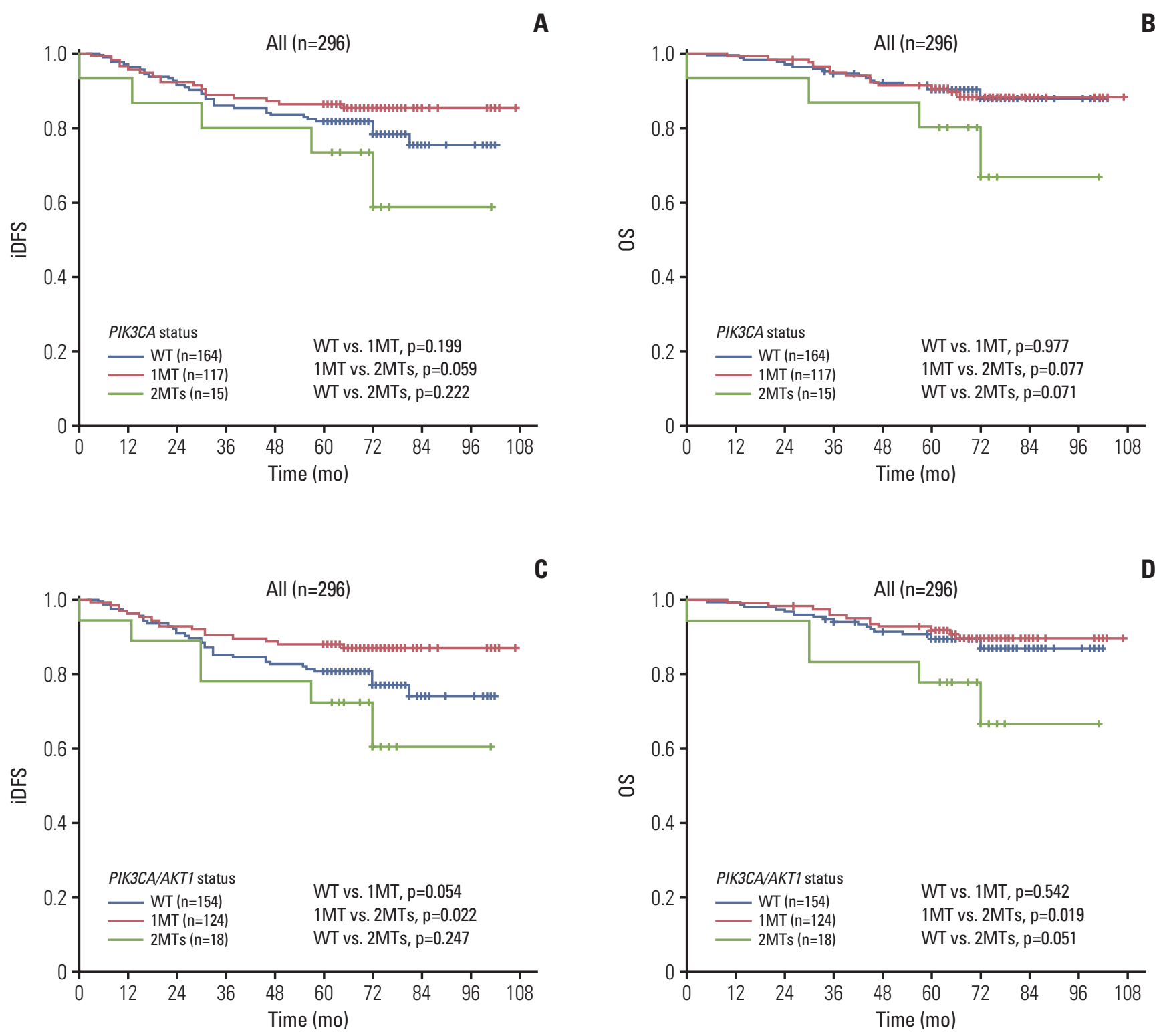

Fig. 1. Impact of PIK3CA/AKT1 mutations on the invasive disease-free survival (iDFS) and overall survival (OS) of 296 breast cancer patients with stage I-III and at least 5-year follow-up. Kaplan-Meier survival analysis; p-values calculated using a log-rank analysis. Estimated iDFS and OS by mutant burden: iDFS (A) and OS (B) for patients with wild-type (WT), one mutation (1MT), and two or three mutations (2MTs) of PIK3CA; iDFS (C) and OS (D) for patients with WT, 1MT, and 2MTs of PIK3CA/AKT1.

(Fig. 1C), the OS of patients with 1MT in PIK3CA/AKT1 was not significantly different than in patients with WT PIK3CA/ AKT1 (Fig. 1D).

\section{2) ER-positive patients (210 cases)}

Regarding the PIK3CA gene, no significant difference in iDFS was observed among ER+ patients with WT, 1MT, and
2MTs in PIK3CA (Fig. 2A). Patients with WT PIK3CA had a trend of better OS than those with 2MTs in PIK3CA (adjusted HR, 0.203; $p=0.058$ ) (Table 3, Fig. 2B). However, there was no difference in OS for patients with WT and $1 \mathrm{MT}$ in PIK3CA (Fig. 2B).

Regarding PIK3CA/AKT1 mutation, ER+ patients with 1MT showed marginally better iDFS (adjusted HR, 0.322; $\mathrm{p}=0.051$ ) and significantly better OS (adjusted HR, 0.195; 
Table 3. Cox multivariate risk model for patients with different mutant burdens (stage I-III and at least 5-year follow-up)

\begin{tabular}{|c|c|c|c|c|c|c|c|c|}
\hline & \multicolumn{4}{|c|}{ PIK3CA mutation } & \multicolumn{4}{|c|}{ PIKЗСА/AKT1 mutation } \\
\hline & \multicolumn{2}{|c|}{ iDFS } & \multicolumn{2}{|l|}{ OS } & \multicolumn{2}{|c|}{ iDFS } & \multicolumn{2}{|l|}{ OS } \\
\hline & HR $(95 \% \mathrm{CI})$ & p-value & HR $(95 \% \mathrm{CI})$ & p-value & HR (95\% CI) & p-value & HR $(95 \% \mathrm{CI})$ & p-value \\
\hline \multicolumn{9}{|l|}{ Total $(n=296)$} \\
\hline 2MTs & $1^{\text {a) }}$ & 0.119 & $1^{\text {a) }}$ & 0.053 & $1^{\mathrm{b})}$ & 0.056 & $1^{\mathrm{b})}$ & 0.008 \\
\hline $1 \mathrm{MT}$ & $\begin{array}{c}0.344 \\
(0.119-0.994)\end{array}$ & 0.049 & $\begin{array}{c}0.222 \\
(0.063-0.787)\end{array}$ & 0.020 & $\begin{array}{c}0.269 \\
(0.098-0.742)\end{array}$ & 0.011 & $\begin{array}{c}0.151 \\
(0.045-0.510)\end{array}$ & 0.002 \\
\hline WT & $\begin{array}{c}0.500 \\
(0.177-1.414)\end{array}$ & 0.191 & $\begin{array}{c}0.221 \\
(0.061-0.799)\end{array}$ & 0.021 & $\begin{array}{c}0.452 \\
(0.169-1.211)\end{array}$ & 0.114 & $\begin{array}{c}0.181 \\
(0.053-0.612)\end{array}$ & 0.006 \\
\hline$E R+(n=210)$ & $1^{c)}$ & 0.402 & $1^{\mathrm{c})}$ & 0.165 & $1^{\text {d) }}$ & 0.125 & $1^{\mathrm{d})}$ & 0.053 \\
\hline $1 \mathrm{MT}$ & $\begin{array}{c}0.444 \\
(0.133-1.482)\end{array}$ & 0.187 & $\begin{array}{c}0.333 \\
(0.073-1.518)\end{array}$ & 0.155 & $\begin{array}{c}0.322 \\
(0.104-1.003)\end{array}$ & 0.051 & $\begin{array}{c}0.195 \\
(0.046-0.820)\end{array}$ & 0.026 \\
\hline WT & $\begin{array}{c}0.588 \\
(0.180-1.921)\end{array}$ & 0.379 & $\begin{array}{c}0.203 \\
(0.039-1.052)\end{array}$ & 0.058 & $\begin{array}{c}0.537 \\
(0.178-1.624)\end{array}$ & 0.271 & $\begin{array}{c}0.167 \\
(0.036-0.774)\end{array}$ & 0.022 \\
\hline HER2 - $(n=210)$ & $1^{\mathrm{e})}$ & 0.105 & $1^{\mathrm{e})}$ & 0.056 & $1^{\mathrm{f})}$ & 0.038 & $1^{\mathrm{f})}$ & 0.017 \\
\hline $1 \mathrm{MT}$ & $\begin{array}{c}0.304 \\
(0.100-0.921)\end{array}$ & 0.035 & $\begin{array}{c}0.246 \\
(0.062-0.972)\end{array}$ & 0.045 & $\begin{array}{c}0.246 \\
(0.084-0.721)\end{array}$ & 0.011 & $\begin{array}{c}0.166 \\
(0.044-0.636)\end{array}$ & 0.009 \\
\hline WT & $\begin{array}{c}0.368 \\
(0.121-1.116)\end{array}$ & 0.077 & $\begin{array}{c}0.171 \\
(0.039-0.741)\end{array}$ & 0.018 & $\begin{array}{c}0.376 \\
(0.131-1.079)\end{array}$ & 0.069 & $\begin{array}{c}0.161 \\
(0.040-0.643)\end{array}$ & 0.010 \\
\hline Ki67 high $(n=210)$ & $1^{\mathrm{g})}$ & 0.027 & $1^{\mathrm{g})}$ & 0.003 & $1^{\mathrm{h})}$ & 0.022 & $1^{\mathrm{h})}$ & 0.002 \\
\hline $1 \mathrm{MT}$ & $\begin{array}{c}0.198 \\
(0.059-0.662)\end{array}$ & 0.009 & $\begin{array}{c}0.096 \\
(0.025-0.372)\end{array}$ & 0.001 & $\begin{array}{c}0.191 \\
(0.057-0.638)\end{array}$ & 0.007 & $\begin{array}{c}0.093 \\
(0.024-0.362)\end{array}$ & 0.001 \\
\hline WT & $\begin{array}{c}0.309 \\
(0.094-1.014)\end{array}$ & 0.053 & $\begin{array}{c}0.114 \\
(0.029-0.446)\end{array}$ & 0.002 & $\begin{array}{c}0.308 \\
(0.094-1.015)\end{array}$ & 0.053 & $\begin{array}{c}0.163 \\
(0.029-0.447)\end{array}$ & 0.002 \\
\hline
\end{tabular}

a) Adjusted by AKT1 mutation, age at diagnosis, menopause at diagnosis, tumor size, lymph node metastasis, estrogen receptor (ER), progesterone receptor (PR), human epidermal growth factor receptor 2 (HER2), Ki67, histological grade, sur-

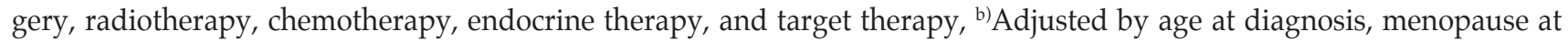
diagnosis, tumor size, lymph node metastasis, ER, PR, HER2, Ki67, histological grade, surgery, radiotherapy, chemotherapy, endocrine therapy, and target therapy, ${ }^{c}$ Adjusted by AKT1 mutation, age at diagnosis, menopause at diagnosis, tumor size, lymph node metastasis, PR, HER2, Ki67, histological grade, surgery, radiotherapy, chemotherapy, endocrine therapy, and target therapy, ${ }^{\text {d) }}$ Adjusted by age at diagnosis, menopause at diagnosis, tumor size, lymph node metastasis, PR, HER2, Ki67, histological grade, surgery, radiotherapy, chemotherapy, endocrine therapy, and target therapy, e)Adjusted by AKT1 mutation, age at diagnosis, menopause at diagnosis, tumor size, lymph node metastasis, ER, PR, Ki67, histological grade, surgery, radiotherapy, chemotherapy, and endocrine therapy, ${ }^{\mathrm{f}}$ Adjusted by age at diagnosis, menopause at diagnosis, tumor size, lymph node metastasis, ER, PR, Ki67, histological grade, surgery, radiotherapy, chemotherapy, and endocrine therapy,

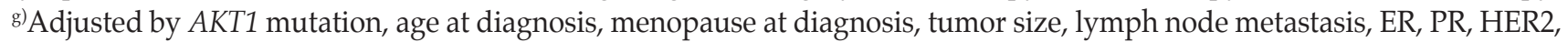
histological grade, surgery, radiotherapy, chemotherapy, endocrine therapy, and target therapy, h)Adjusted by age at diagnosis, menopause at diagnosis, tumor size, lymph node metastasis, ER, PR, HER2, histological grade, surgery, radiotherapy, chemotherapy, endocrine therapy, and target therapy.

$\mathrm{p}=0.026)$ than those with 2MTs (Table 3, Fig. 2C and D). Patients with WT PIK3CA/AKT1 exhibited better OS than those with 2MTs in PIK3CA/AKT1 (adjusted HR, 0.167; $\mathrm{p}=0.022$ ) (Table 3, Fig. 2D), but no significant difference in iDFS (Fig. 2C). The iDFS and OS of patients with 1MT were not significantly different from those with WT PIK3CA/AKT1 (Fig. 2C and D).

\section{3) HER2-negative patients ( 210 cases)}

Regarding PIK3CA mutation, HER2- patients with 1MT exhibited significantly better iDFS (adjusted HR, 0.304; $\mathrm{p}=0.035$ ) and OS (adjusted HR, 0.246; $\mathrm{p}=0.045$ ) than those with 2MTs (Table 3, Fig. 3A and B). Patients with WT PIK3CA had better OS (adjusted HR, 0.171; $\mathrm{p}=0.018$ ) and showed a trend toward better iDFS (adjusted HR, 0.368; 

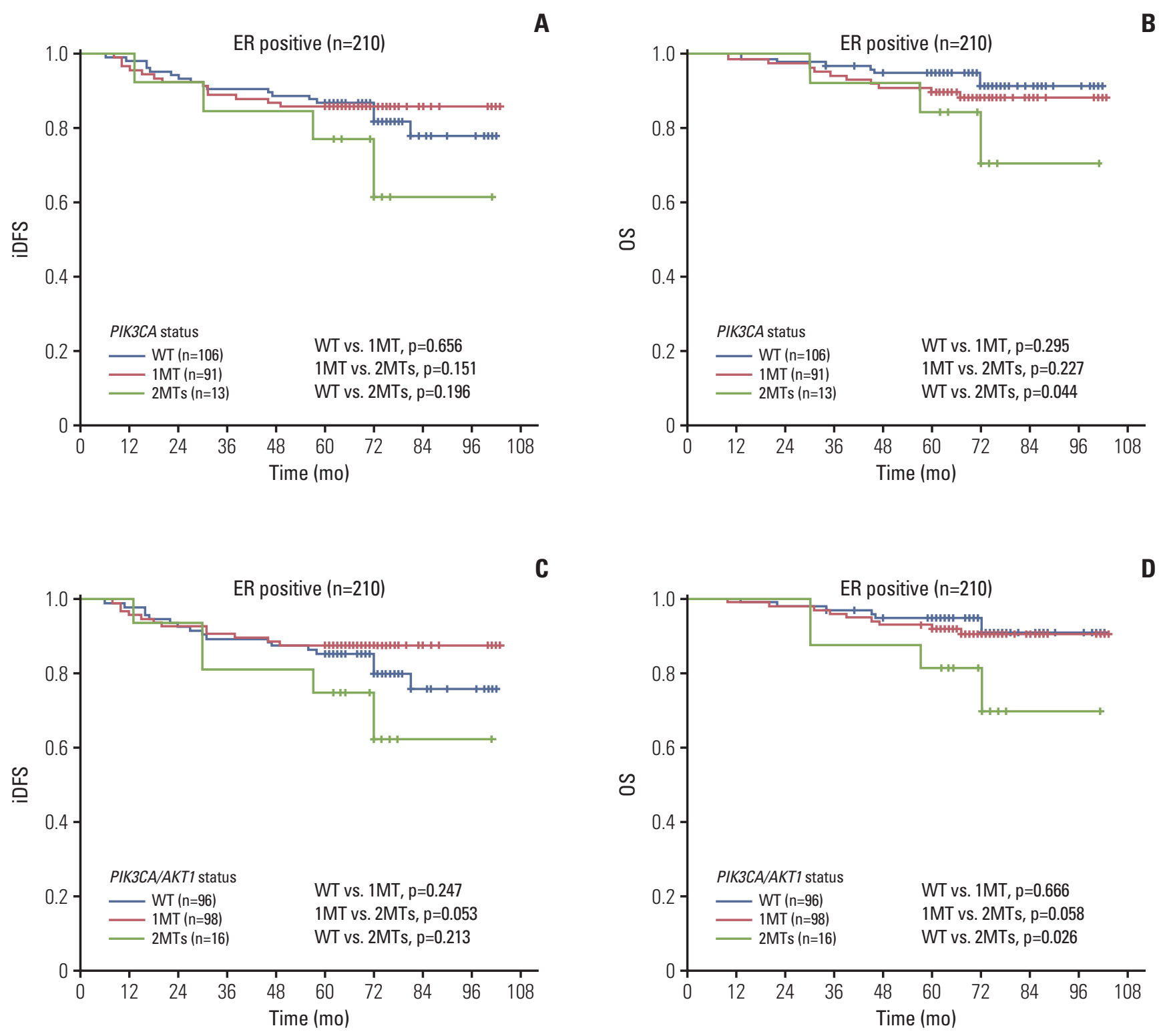

Fig. 2. Impact of $P I K 3 C A / A K T 1$ mutations on the invasive disease-free survival (iDFS) and overall survival (OS) of 210 estrogen receptor (ER) positive breast cancer patients with stage I-III and at least 5-year follow-up. Kaplan-Meier survival analysis; p-values calculated using a log-rank analysis. Estimated iDFS and OS by mutant burden: iDFS (A) and OS (B) for patients with wild-type (WT), one mutation (1MT), and two or three mutations (2MTs) of PIK3CA; iDFS (C) and OS (D) for patients with WT, 1MT, and 2MTs of PIK3CA/AKT1.

$\mathrm{p}=0.077$ ) than those with 2MTs in PIK3CA (Table 3, Fig. 3A and $\mathrm{B})$. No difference in iDFS or OS was observed between patients with WT and $1 \mathrm{MT}$ in PIK3CA (Fig. 3A and B).

Regarding PIK3CA/AKT1 mutation, HER2- patients with 1MT had better iDFS (adjusted HR, 0.246; $\mathrm{p}=0.011$ ) and OS (adjusted HR, 0.166; $\mathrm{p}=0.009$ ) than those with 2MTs (Table 3, Fig. 3C and D). Patients with WT PIK3CA/AKT1 had better OS (adjusted HR, 0.161; $\mathrm{p}=0.010$ ) and showed a trend toward better iDFS (adjusted HR, 0.376; $\mathrm{p}=0.069$ ) than those with 2MTs (Table 3, Fig. 3C and D). There was no difference in iDFS and OS between patients with WT and $1 \mathrm{MT}$ in PIK3CA/ AKT1.

\section{4) Patients with high expression of Ki67 (240 cases)}

Regarding PIK3CA mutation, patients with high expres- 

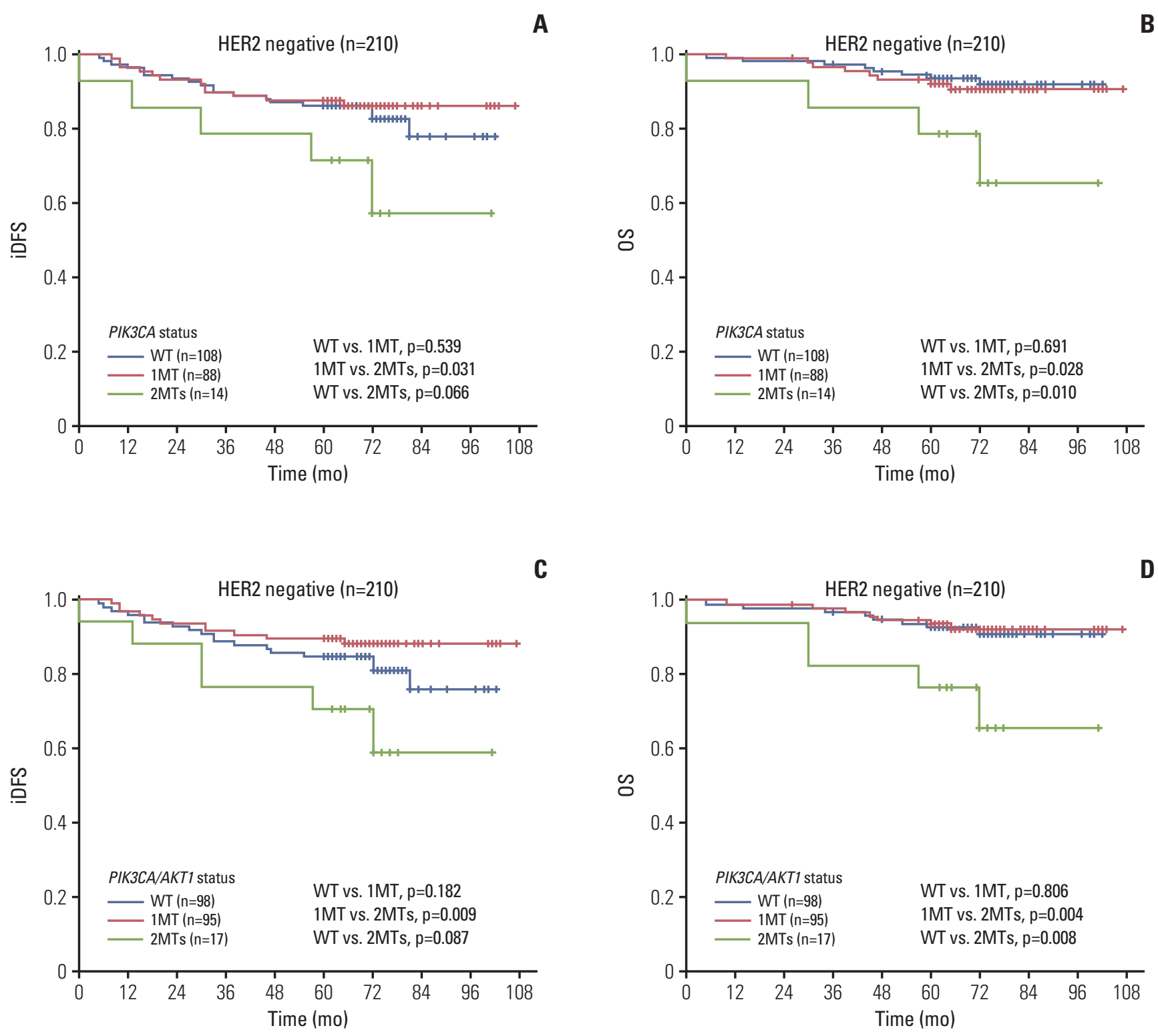

Fig. 3. Impact of PIK3CA/AKT1 mutations on the invasive disease-free survival (iDFS) and overall survival (OS) of 210 human epidermal growth factor receptor 2 (HER2) negative breast cancer patients with stage I-III and at least 5-year followup. Kaplan-Meier survival analysis; p-values calculated using a log-rank analysis. Estimated iDFS and OS by mutant burden: iDFS (A) and OS (B) for patients with wild-type (WT), one mutation (1MT), and two or three mutations (2MTs) of PIK3CA; iDFS (C) and OS (D) for patients with WT, 1MT, and 2MTs of PIK3CA/AKT1.

sion of Ki67 and 1MT had better iDFS (adjusted HR, 0.198; $\mathrm{p}=0.009$ ) and $\mathrm{OS}$ (adjusted HR, 0.096; $\mathrm{p}=0.001$ ) than those with 2MTs (Table 3, Fig. 4A and B). Patients with WT PIK3CA exhibited better OS than those with 2MTs in PIK3CA (adjusted HR, 0.114; $\mathrm{p}=0.002$ ) (Table 3, Fig. 4B), with no significant difference in iDFS. The iDFS and OS of patients with 1MT were not significantly different than in patients with WT PIK3CA (Fig. 4A and B).
Regarding PIK3CA/AKT1 mutation, patients with 1MT had better iDFS (adjusted HR, 0.191; $\mathrm{p}=0.007$ ) and OS (adjusted HR, 0.093; $p=0.001$ ) than those with 2MTs (Table 3, Fig. 4C and D). Patients with WT PIK3CA/AKT1 exhibited better OS (adjusted HR, 0.163; $\mathrm{p}=0.002$ ) and a trend toward better iDFS (adjusted HR, 0.308; $\mathrm{p}=0.053$ ) than those with 2MTs (Table 3, Fig. $4 \mathrm{C}$ and D). No difference in iDFS or OS was observed between patients with 1MT and WT PIK3CA/AKT1. 

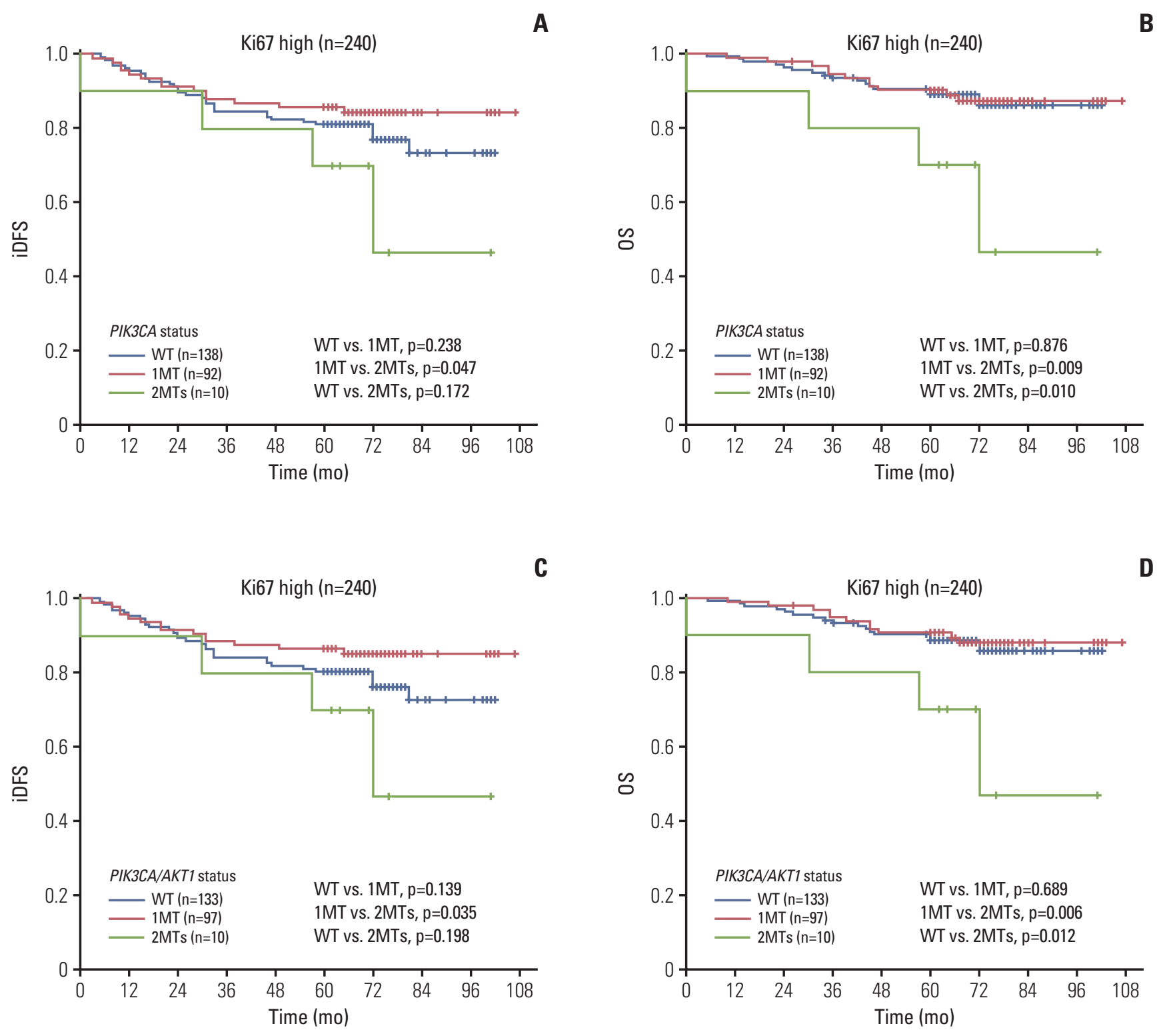

Fig. 4. Impact of PIK3CA/AKT1 mutations on the invasive disease-free survival (iDFS) and overall survival (OS) of 240 Ki67 high breast cancer patients with stage I-III and at least 5-year follow-up. Kaplan-Meier survival analysis; p-values calculated using a log-rank analysis. Estimated iDFS and OS by mutant burden: iDFS (A) and OS (B) for patients with wild-type (WT), one mutation (1MT), and two or three mutations (2MTs) of PIK3CA; iDFS (C) and OS (D) for patients with WT, 1MT, and 2MTs of PIK3CA/AKT1.

The prognosis between patients with mutant (hotspot and non-hotspot) and WT PIK3CA genes was also compared. Results showed that the iDFS and OS of patients with mutant PIK3CA was not significantly different from patients with WT PIK3CA, whether they were in the entire group or one of the three subgroups (ER+, HER2-, Ki67 high) (S4-S7 Figs.). Survival was also evaluated among patients with hotspot MT, non-hotspot MT and WT PIK3CA, and there was no dif- ference in iDFS or OS (S4-S7 Figs.).

Survival between patients with mutant and WT AKT1 was also evaluated, and there was no difference in iDFS or OS, whether they were in the entire group or one of the 3 subgroups (S8 Fig.). In addition, we compared iDFS and OS between patients with mutant (2MTs plus 1MT) or WT PIK3CA/AKT1, and no significant difference was observed (S9 Fig.). 


\section{Discussion}

This study provided new insight into the complexity of PIK3CA and AKT1 mutations in Chinese breast cancer patients. AKT1 mutations were detected in $3.6 \%$ of the 507 patients, and $4.9 \%(18 / 370)$ in the luminal subtype, which is similar to the $3.14 \%$ in the TCGA report [3]. PIK3CA mutations were detected in $46.5 \%$ of the 507 patients, and the hotspot mutations of PIK3CA were $39.4 \%$. The frequency of PIK3CA mutations was $50.8 \%(188 / 370)$ in the luminal subtype, $41.8 \%$ (28/67) in the HER2-positive subtype, and 28.6\% $(20 / 70)$ in triple-negative breast cancer. This was similar to the PIK3CA mutation rates of BOLERO-2 trial, which was also detected via NGS [15]. In contrast, this was significantly higher than the PIK3CA mutation rates previously reported in a cohort of 729 Chinese women with breast cancer (28.3\%) [11], as well as what was reported in a cohort of HER2amplified metastatic breast cancer (24\%) [14], which only detected hotspot mutations of PIK3CA. These results illustrated that NGS is a reliable method that can detect PIK3CA and $A K T 1$ mutations efficiently.

Also, we confirmed significant associations of AKT1 mutations with clinicopathological and molecular characteristics, as well as PIK3CA mutations (whether hotspot or nonhotspot mutations). Both AKT1 mutations and PIK3CA mutations were significantly associated with ER positive, PR positive and HER2 negative. AKT1 mutations were also associated with high Ki67 expression. But Ki67 expression was not different among patients harboring hotspot mutation, non-hotspot mutation, or WT PIK3CA. This is consistent with the literature $[6,7,12,21]$.

The association between PIK3CA mutations and prognosis has been evaluated by many studies. However, results from these studies are still inconclusive. Some studies showed favorable $[6,7,22]$, and others showed adverse $[4,6,10,14$, 23-25], impacts of PIK3CA mutations on patient outcomes, while a significant number of reports did not show any prognostic significance [5,8,9,11-13,26]. In HER2-positive breast cancers, PIK3CA mutation was associated to poor prognosis in several studies $[4,25,27]$, while the association of PIK3CA mutation with prognosis was not significantly in the FinHER trial [5]. PIK3CA mutation predicted poorer pathologic complete response $(\mathrm{pCR})$ in patients with HER2+ breast cancer treated with neoadjuvant therapies $[8,9,11,28]$; however, the association of PIK3CA mutation with $\mathrm{pCR}$ did not translate into DFS or OS outcomes $[29,30]$. In the present study, patients with 1MT of the PIK3CA gene did not have significantly different iDFS or OS when compared to those with WT PIK3CA, whether they were in the entire group or all three subgroups (ER+, HER2-, Ki67 high). In addition, PIK3CA mutation was not associated with prognosis of
HER2-positive patients (WT vs. MT, $\mathrm{P}_{\mathrm{DFS}}=0.268, \mathrm{P}_{\mathrm{OS}}=0.842$, data not shown). These results demonstrated that single PIK3CA gene mutation might have little effect on the prognosis of breast cancer patients.

Preclinical models found PIK3CA mutations alone, compared to PTEN loss or AKT1 mutations, can cause weaker or more inconsistent activation of PI3K-AKT signaling [31,32]. If this signaling pathway accumulates more genetic alteration burden, it may lead to higher activation of the pathway. For prognosis, patients simultaneously harboring both PIK3CA mutation and PTEN loss had lower DFS and OS [12] which suggest that it may be possible to analyze the prognosis of patients according to the genetic alteration burden of PI3K-AKT pathway. In the present study, we compared prognosis of patients with $2 \mathrm{MT}$ of PIK3CA/AKT1 to those with $1 \mathrm{MT}$ or wild-type PIK3CA/AKT1, which showed 2MT of PIK3CA/AKT1 caused poorer iDFS and OS. Patients with 2MT of PIK3CA also had poorer prognosis. These results suggested that greater number of alterations in PI3K/AKT/ mTOR pathway might cause higher activation of this pathway, which was associated with poor prognosis.

Drugs targeting the PI3K / AKT / mTOR pathway in breast cancer are widely known, and some of them are approved for use in ER+ advanced breast cancer. Several studies have attempted to determine in which patients these drugs would achieve better clinical efficacy. An exploratory analysis using tumor samples from the BOLERO-2 trial showed that the efficacy of everolimus was largely independent of the most commonly altered genes (PIK3CA, FGFR1 and CCND1) in hormone receptor-positive and HER2-negative breast cancer. Subgroup analysis showed that PIK3CA exon-9 mutations were associated with a greater benefit from everolimus than exon-20 mutations [15]. However, another study using cellfree DNA from plasma samples from the BOLERO-2 trial showed that everolimus prolonged median progression-free survival in patients with PIK3CA H1047R and E545K/E542K mutations to a similar degree [33]. These different results demonstrate that more accurate and effective biomarkers are still needed to determine the efficacy of drugs targeting the PI3K/AKT/mTOR pathway.

There are some limitations to the present study. This was a single-center retrospective study with a relatively small sample size. Although the present study found that a tumor mutational burden of 2-3 in PIK3CA/AKT1 was associated with poorer outcomes, the sample size in this subgroup was relatively small compared to patients with WT or 1MT in PIK3CA/AKT1. The active burden for the entire PI3K / AKT1/ mTOR pathway should be evaluated in a larger sample with a longer follow-up time.

In summary, PIK3CA/AKT1 mutation was detected using NGS in $49.3 \%$ of breast cancer patients from a single hospital in West China, with the highest mutation frequency in the 
luminal cancers. PIK3CA/AKT1 mutation was associated with positive ER, positive PR, or negative HER2 expression status. The prognosis of patients with one mutation in PIK3CA (or PIK3CA/AKT1) was not significantly different than in WT patients. Patients with a tumor mutational burden of 2-3 in PIK3CA (or PIK3CA/AKT1) had poorer prognosis in the entire group, or all 3 subgroups (ER+, HER2-, Ki67 high), particularly with respect to OS. These findings suggest that, in addition to mutation frequency, the tumor mutational burden of the PIK3CA and AKT1 genes should also be considered, and that these could be potential prognostic biomarkers in breast cancer patients.

\section{Electronic Supplementary Material}

Supplementary materials are available at Cancer Research and Treatment website (https://www.e-crt.org).

\section{Conflicts of Interest}

The authors have read the journal's policy and have the following competing interests: Xuehua Zhu, Yun Sun, Jiemin Wang and Min Hu were employees of Dizal Pharmaceutical during this study. The remaining authors declare that they have no competing interests. This does not alter the authors' adherence to Cancer Research and Treatment policies on sharing data and materials.

This work was supported by Dizal Pharmaceutical (Recipient: Z.H.). The funder provided support in the form of salaries for authors (X.H.Z., Y.S., J.M.W., and M.H.), but did not have any additional role in the study design, data collection and analysis, decision to publish, or preparation of the manuscript.

\section{Acknowledgments}

We would like to thank LetPub (www.letpub.com) for providing linguistic assistance during the preparation of this manuscript.

\section{References}

1. Rodon J, Dienstmann R, Serra V, Tabernero J. Development of PI3K inhibitors: lessons learned from early clinical trials. Nat Rev Clin Oncol. 2013;10:143-53.

2. Thorpe LM, Yuzugullu H, Zhao JJ. PI3K in cancer: divergent roles of isoforms, modes of activation and therapeutic targeting. Nat Rev Cancer. 2015;15:7-24.

3. Cancer Genome Atlas Network. Comprehensive molecular portraits of human breast tumours. Nature. 2012;490:61-70.

4. Cizkova M, Dujaric ME, Lehmann-Che J, Scott V, Tembo O, Asselain B, et al. Outcome impact of PIK3CA mutations in HER2-positive breast cancer patients treated with trastuzumab. Br J Cancer. 2013;108:1807-9.

5. Loi S, Michiels S, Lambrechts D, Fumagalli D, Claes B, Kellokumpu-Lehtinen PL, et al. Somatic mutation profiling and associations with prognosis and trastuzumab benefit in early breast cancer. J Natl Cancer Inst. 2013;105:960-7.

6. Cizkova M, Vacher S, Meseure D, Trassard M, Susini A, Mlcuchova D, et al. PIK3R1 underexpression is an independent prognostic marker in breast cancer. BMC Cancer. 2013;13: 545.

7. Sabine VS, Crozier C, Brookes CL, Drake C, Piper T, van de Velde CJ, et al. Mutational analysis of PI3K/AKT signaling pathway in tamoxifen exemestane adjuvant multinational pathology study. J Clin Oncol. 2014;32:2951-8.

8. Loibl S, von Minckwitz G, Schneeweiss A, Paepke S, Lehmann A, Rezai M, et al. PIK3CA mutations are associated with lower rates of pathologic complete response to anti-human epidermal growth factor receptor 2 (her2) therapy in primary HER2overexpressing breast cancer. J Clin Oncol. 2014;32:3212-20.

9. Majewski IJ, Nuciforo P, Mittempergher L, Bosma AJ, Eidt- mann $\mathrm{H}$, Holmes E, et al. PIK3CA mutations are associated with decreased benefit to neoadjuvant human epidermal growth factor receptor 2-targeted therapies in breast cancer. J Clin Oncol. 2015;33:1334-9.

10. Deng L, Chen J, Zhong XR, Luo T, Wang YP, Huang HF, et al. Correlation between activation of $\mathrm{PI} 3 \mathrm{~K} / \mathrm{AKT} / \mathrm{mTOR}$ pathway and prognosis of breast cancer in Chinese women. PLoS One. 2015;10:e0120511.

11. Yuan H, Chen J, Liu Y, Ouyang T, Li J, Wang T, et al. Association of PIK3CA mutation status before and after neoadjuvant chemotherapy with response to chemotherapy in women with breast cancer. Clin Cancer Res. 2015;21:4365-72.

12. Papaxoinis G, Kotoula V, Alexopoulou Z, Kalogeras KT, Zagouri F, Timotheadou E, et al. Significance of PIK3CA mutations in patients with early breast cancer treated with adjuvant chemotherapy: a Hellenic Cooperative Oncology Group (HeCOG) Study. PLoS One. 2015;10:e0140293.

13. Engels CC, Kiderlen M, Bastiaannet E, van Eijk R, Mooyaart A, Smit VT, et al. The clinical value of HER-2 overexpression and PIK3CA mutations in the older breast cancer population: a FOCUS study analysis. Breast Cancer Res Treat. 2016;156:3 61-70.

14. Xu B, Guan Z, Shen Z, Tong Z, Jiang Z, Yang J, et al. Association of phosphatase and tensin homolog low and phosphatidylinositol 3-kinase catalytic subunit alpha gene mutations on outcome in human epidermal growth factor receptor 2-positive metastatic breast cancer patients treated with first-line lapatinib plus paclitaxel or paclitaxel alone. Breast Cancer Res. 2014;16:405.

15. Hortobagyi GN, Chen D, Piccart M, Rugo HS, Burris HA 3rd, 
Pritchard KI, et al. Correlative analysis of genetic alterations and everolimus benefit in hormone receptor-positive, human epidermal growth factor receptor 2-negative advanced breast cancer: Results From BOLERO-2. J Clin Oncol. 2016;34:419-26.

16. Pang B, Cheng S, Sun SP, An C, Liu ZY, Feng X, et al. Prognostic role of PIK3CA mutations and their association with hormone receptor expression in breast cancer: a meta-analysis. Sci Rep. 2014;4:6255.

17. Guideline Recommendations for Immunohistochemistry Detection in Breast Cancer Group. Guideline for testing of estrogen and progesterone receptors in breast cancer. Zhonghua Bing Li Xue Za Zhi. 2015;44:237-9.

18. Guideline Recommendations for HER2 Detection in Breast Cancer Group. Guidelines for HER2 detection in breast cancer, the 2014 version. Zhonghua Bing Li Xue Za Zhi. 2014;43: 262-7.

19. Goldhirsch A, Winer EP, Coates AS, Gelber RD, Piccart-Gebhart M, Thurlimann B, et al. Personalizing the treatment of women with early breast cancer: highlights of the St Gallen International Expert Consensus on the Primary Therapy of Early Breast Cancer 2013. Ann Oncol. 2013;24:2206-23.

20. Gourgou-Bourgade S, Cameron D, Poortmans P, Asselain B, Azria D, Cardoso F, et al. Guidelines for time-to-event end point definitions in breast cancer trials: results of the DATECAN initiative (Definition for the Assessment of Time-toevent Endpoints in CANcer trials). Ann Oncol. 2015;26:2505-6.

21. Ahmad F, Badwe A, Verma G, Bhatia S, Das BR. Molecular evaluation of PIK3CA gene mutation in breast cancer: determination of frequency, distribution pattern and its association with clinicopathological findings in Indian patients. Med Oncol. 2016;33:74.

22. Takeshita T, Yamamoto $Y$, Yamamoto-Ibusuki M, Inao T, Sueta A, Fujiwara S, et al. Prognostic role of PIK3CA mutations of cell-free DNA in early-stage triple negative breast cancer. Cancer Sci. 2015;106:1582-9.

23. Leo F, Bartels S, Magel L, Framke T, Busche G, Jonigk D, et al. Prognostic factors in the myoepithelial-like spindle cell type of metaplastic breast cancer. Virchows Arch. 2016;469:191-201.

24. Jacot W, Mollevi C, Fina F, Lopez-Crapez E, Martin PM, Colombo PE, et al. High EGFR protein expression and exon 9 PIK3CA mutations are independent prognostic factors in triple negative breast cancers. BMC Cancer. 2015;15:986.

25. Baselga J, Cortes J, Im SA, Clark E, Ross G, Kiermaier A, et al. Biomarker analyses in CLEOPATRA: a phase III, placebo-controlled study of pertuzumab in human epidermal growth factor receptor 2-positive, first-line metastatic breast cancer. J Clin Oncol. 2014;32:3753-61.

26. Kim JY, Lee E, Park K, Park WY, Jung HH, Ahn JS, et al. Clinical implications of genomic profiles in metastatic breast cancer with a focus on TP53 and PIK3CA, the most frequently mutated genes. Oncotarget. 2017;8:27997-8007.

27. Jensen JD, Knoop A, Laenkholm AV, Grauslund M, Jensen MB, Santoni-Rugiu E, et al. PIK3CA mutations, PTEN, and pHER2 expression and impact on outcome in HER2-positive early-stage breast cancer patients treated with adjuvant chemotherapy and trastuzumab. Ann Oncol. 2012;23:2034-42.

28. Ibrahim EM, Kazkaz GA, Al-Mansour MM, Al-Foheidi ME. The predictive and prognostic role of phosphatase phosphoinositol-3 (PI3) kinase (PIK3CA) mutation in HER2-positive breast cancer receiving HER2-targeted therapy: a meta-analysis. Breast Cancer Res Treat. 2015;152:463-76.

29. Loibl S, Majewski I, Guarneri V, Nekljudova V, Holmes E, Bria $\mathrm{E}$, et al. PIK3CA mutations are associated with reduced pathological complete response rates in primary HER2-positive breast cancer: pooled analysis of 967 patients from five prospective trials investigating lapatinib and trastuzumab. Ann Oncol. 2016;27:1519-25.

30. Yang SX, Polley E, Lipkowitz S. New insights on PI3K/AKT pathway alterations and clinical outcomes in breast cancer. Cancer Treat Rev. 2016;45:87-96.

31. Oda K, Okada J, Timmerman L, Rodriguez-Viciana P, Stokoe D, Shoji K, et al. PIK3CA cooperates with other phosphatidylinositol 3'-kinase pathway mutations to effect oncogenic transformation. Cancer Res. 2008;68:8127-36.

32. Stemke-Hale K, Gonzalez-Angulo AM, Lluch A, Neve RM, Kuo WL, Davies M, et al. An integrative genomic and proteomic analysis of PIK3CA, PTEN, and AKT mutations in breast cancer. Cancer Res. 2008;68:6084-91.

33. Moynahan ME, Chen D, He W, Sung P, Samoila A, You D, et al. Correlation between PIK3CA mutations in cell-free DNA and everolimus efficacy in HR(+), HER2(-) advanced breast cancer: results from BOLERO-2. Br J Cancer. 2017;116:726-30. 